\title{
Superficial Temporal Artery-Middle Cerebral Artery Bypass (STA-MCA) for Middle Cerebral Artery Dissecting Aneurysm in a Child
}

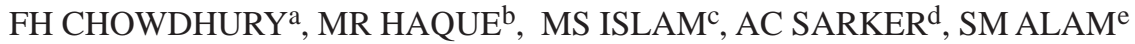

\begin{abstract}
Intracranial arterial dissection or dissecting aneurysm is relatively uncommon and usually involve the vertebrobasilar system. Here we report a pediatric case of middle cerebral artery dissection/dissecting aneurysm with sub arachnoid hemorrhage and Sylvian fissure hematoma on dominant side. After initial evacuation of hematoma, we went for trapping and excision of dissection/dissecting
\end{abstract}

\section{Introduction:}

Intracranial aneurysms may be saccular and fusiform types. Fusiform aneurysms are nonsaccular dilatations involving the entire vessel wall for a short distance $\mathrm{e}^{1,2,3}$ usually caused by dissection or atherosclerosis ${ }^{4}$. The underlying pathologies are disorders of collagen and elastin metabolism, infections and-very rarely due to neoplastic invasion of the arterial wall ${ }^{5}$. Fusiform aneurysms have different underlying pathologies, hemodynamics, anatomical distributions, natural histories and treatments than do the saccular variety ${ }^{4}$. Clinical presentation is due to occlusion, arterial rupture, or a pressure effect.

Intracranial fusiform aneurysms are rare and represents only $3 \%-13 \%$ of all intracranial aneurysms ${ }^{1}$ and are

a. Dr. Forhad Hossain Chowdhury, Neurosurgeon, Dept. of Neurosurgery, Dhaka Medical College Hospital, Dhaka.

b. Dr. Md. Raziul Haque, Associate Professor, Dept. of Neurosurgery, Dhaka Medical College Hospital, Dhaka.

c. Dr. Md. Shafiqul Islam, Neurosurgeon, Dept. of Neurosurgery, Dhaka Medical College Hospital, Dhaka.

d. Dr Asit Chandra Sarker, Associate Professor, Dept. of Neurosurgery, Dhaka Medical College Hospital, Dhaka.

e. Dr. Sarwar Morshed Alam, Dept. of Neurosurgery, Dhaka Medical College Hospital, Dhaka.

Address of Correspondence: Dr. Forhad Hossain Chowdhury, Flat No-C6, Rupayan Angel,152 Shantinagar, Dhaka, Bangladesh. Phone+8801711949570, e-mail- forhadchowdhury74@yahoo.com Received: 1 January, 2013

Accepted: 15 January, 2014 aneurysm followed by STA-MCA bypass. Patient recovered fully from her neurological deficit. Probably this is the first reported case of STA-MCA bypass in Bangladesh.

Key Words: STA-MCA bypass, Middle cerebral artery dissecting aneurysm, Intracranial fusiform aneurysm.

(J Banagladesh Coll Phys Surg 2014; 32: 94-98)

usually located in the vertebro-basilar system ${ }^{3,6}$. Fusiform aneurysms in the anterior circulation remain rare and occur mostly in the middle cerebral artery and internal carotid artery ${ }^{4}$. There are some sporadic case reports about the treatment of fusiform aneurysms ${ }^{1,4,5}$ Here we report a pediatric case of middle cerebral artery dissecting aneurysm on dominant side. After initial evacuation of hematoma, we went for trapping and excision of dissection/dissecting aneurysm followed by STA-MCA bypass.

\section{Case Report:}

A thirteen years old right handed girl presented with history of headache, vomiting, alteration of level of consciousness and right sided paralysis. On admission her GCS score was E2V2M5=09 with right sided hemiplegia (MRC grade-0). Emergency CT scan showed left Sylvian fissure, insula and external capsular zone hematoma with mass effect (Figure1A,B\&C). Through left temporo-parietal craniotomy hematoma was evacuated. After operation her GCS improved a little (E4V2M5=11) and remained obtunded. Her hemiplegia remained unchanged. We did CT angiogram of brain that showed a left sided M3 fusiform aneurysm (Figure2A,B\&C). Cerebral DSA failed to show the aneurysm and distal part of involved M3 and M4.But involved M3 and M4 was visualized lately by retrograde flow from cortical anastomosis (Figure2D). 
We re-operated on her 10 days after hematoma evacuation through the previously done craniotomy site. After dural opening posterior ramus of Sylvian fissure was exposed by arachnoid dissection. Insula, M3 and M4 portions of MCA with aneurysm were identified. Thrombosed fusiform aneurysm of M3 was found in posterior part of Sylvian fissure. We trapped and excised the aneurysm (there was some retrograde flow at the distal part of the aneurysm) (Fig. 3A). Then by using frontal branch of STA (which was already dissected out and fashioned for microvascular anastomosis) a STAMCA bypass was made with in the involved distal M3 segment of MCA (Fig. 3B) using high magnification under operating microscope. Dura was loosely closed around the STA. Wound was closed in layers without any drain.

On the first POD she became fully conscious with GCS score-15. From $3^{\text {rd }}$ post operative day (POD) her hemiplegia began to improve (Fig. 4A). At the end of two week she could walk with support and at the end of three week she could walk without support. At the time of discharge her higher psychic functions including speech were normal and there was no nominal dysphasia. At the end of three month after operation she returned to her school. Histopathology report of excised aneurysm wall failed to give any specific vessel wall disorder. Postoperative MRA of brain (six month after operation) showed patent STA-MCA bypass (Fig. 4B)

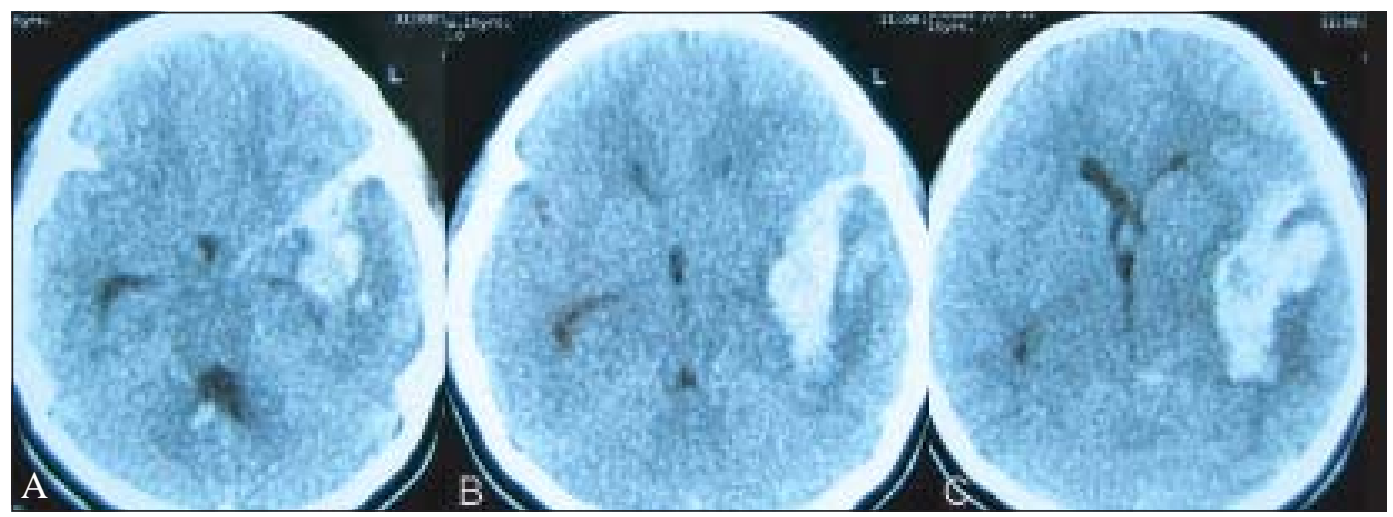

Fig.-1: Emergency CT scan of brain, serial axial sections $(A, B \& C)$ showing left Sylvian fissure, insula and external capsular zone hematoma with mass effect.

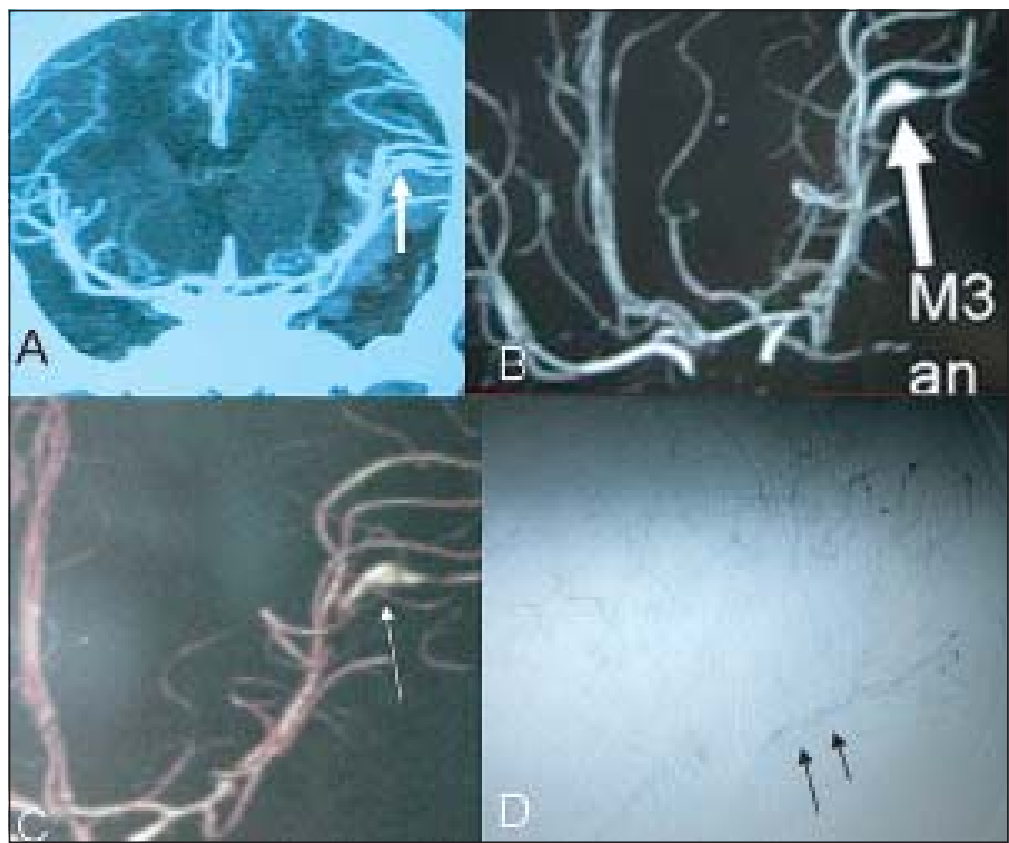

Fig.-2 (A, B \& C): CT angiogram of brain showing left sided M3 dissecting aneurysm(arrow marked). (D) Cerebral DSA showing slow and late retrograde filling of involved left distal M3 and M4 (arrows marked) form cortical anastomosis. 


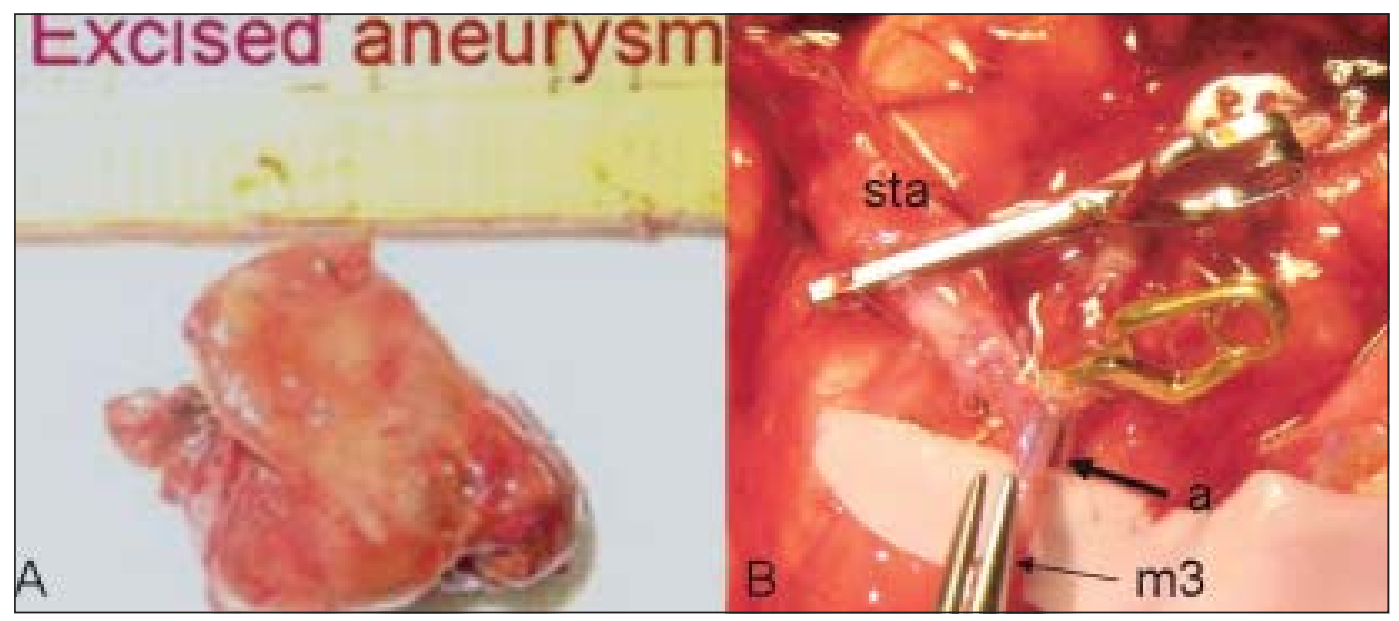

Fig.-3: Peroperative pictures. A-excised dissecting aneurysm, B-STA-MCA (involved distal M3) bypass.

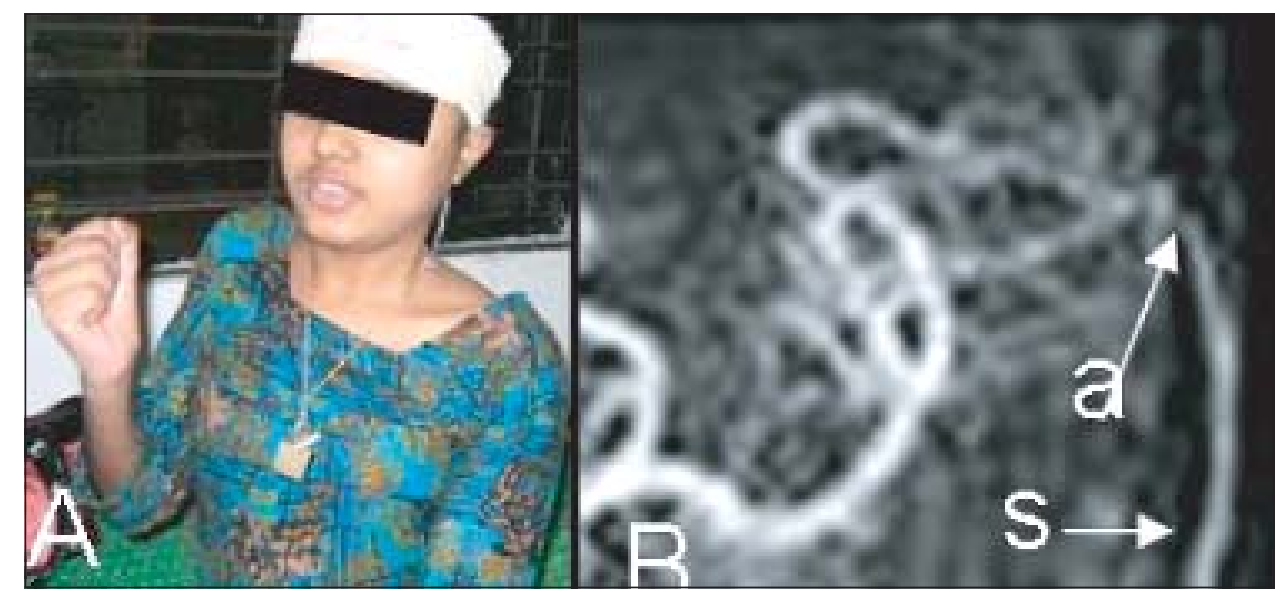

Fig.-4:A-postoperative picture of patient in recovering stage of right sided hemiplegia. B-MRA of brain( 6 months after operation) patient left STA-MCA bypass(a-anastomosis\& STA)

\section{Discussion:}

All fusiform aneurysms are spindle shape when viewed from outside ${ }^{4,5}$. Conceptually, there is still confusion as to the etiological, clinical and radiological features of fusiform aneurysms. However, the classic dissecting aneurysm also has a fusiform appearance ${ }^{5}$ and several authors have recently reported the presence of fusiform aneurysms caused by dissection ${ }^{1-4,7-10}$. Spontaneous fusiform aneurysms are more often found in younger patients $1,2,3,4,10,11$ and are more frequent in men ${ }^{4,11}$.

Dissecting aneurysm can progress from a small focal dilatation or vessel narrowing, to a relatively thickwalled, tortuous dilatation and elongation of the artery.
Hemorrhage is the most common presentation in patients with small lesions with focal dilatation, whereas ischemic symptoms were the most common presentation of patients with stenosis or vascular occlussion ${ }^{5}$. In our case initial symptoms were due to hemorrhage but later it could be assumed that symptoms were also due to ischaemia from thrombotic occlusion of the involved artery. Here during operation we found retrograde flow from involved artery distal to aneurysm. That means supplying area of that artery was just getting adequate arterial flow ${ }^{1}$ ' for survival but not adequate for functioning through cortical anastomosis. STA-MCA bypass reinforced the arterial supply of the brain to recover its functions. 
Dissection has been proposed as the main underlying cause of fusiform aneurysms and most commonly involves the posterior circulation, especially vertebral and basilar arteries $3,4,7,8,10,11$ Dissecting aneurysms can originate in any regions of the anterior circulation, such as the $\mathrm{ICA}^{5}$, MCA ${ }^{1,2,4,8,10}$ the ACA $7,8,9,11$ and rarely in the anterior choroidal artery ${ }^{5}$. The MCA is the most common $^{4,12}$. Various etiological factors for fusiform aneurysms have been proposed, including atherosclerosis, vessel dissection and association with other diseases such as von Recklinghausen's disease, fibromuscular dysplasia, systemic lupus erythematosus and various collagen-associated vascular diseases 2,4,12 . An intramural thrombus that ruptures into the lumen will cause a distal embolization and further expansion of the intramural clot will lead to vessel occlusion ${ }^{4,5}$. After occluding vessel by intramural clot, it can be recanalized and enlarged the dissection both laterally and longitudinally. Serpentine channel forms as disease extends longitudinally, combined with varying degrees of intraluminal thrombosis. Treatment of fusiform aneurysms should be based on the presence and type of symptoms, the lesion size and location and the risk of any accompanying intervention. Day et al. ${ }^{4}$ have suggested guidelines for the treatment of patients with dissecting aneurysms of the MCA. They recommend that most small and some large focal dilatations, especially those that are asymptomatic, should be treated conservatively unless serial neuroimaging assessment indicates significant enlargement over time. However, the appearance of symptoms requires aggressive intervention. Lanzino et al. ${ }^{9}$ and Nikawa et al. ${ }^{10}$ also recommended conservative treatment in patients with dissecting aneurysms without neurological deterioration or recurrent SAH because of the possibility of spontaneous evolution of a dissecting aneurysm.

Day et al. ${ }^{4}$ and several other authors ${ }^{6,7,9,11}$ recommend that patients with stenotic or occlusive lesions presenting with acute ischemic symptoms should be treated conservatively. However, Kurino et al. ${ }^{13}$ reported a patient with a dissecting aneurysm in the MCA who presented with ischemic symptoms and who showed a poor outcome after conservative treatment. They recommend surgical revascularization distal to the compromised artery. Consideration of aggressive treatment with endovascular or surgical methods for focal dilating fusiform aneurysms is recommended. ${ }^{5}$ Several authors ${ }^{4,10}$ recommended a proximal occlusion or trapping with or without resection combined with end-to-end anastomosis or EC-IC bypass 2,3,4,7,8,9,11,12,14. But some can be treated using encircled aneurysm clips, such as the Sundt clip, by partial clipping followed by wrapping if the aneurysm is not ruptured case, and by occlusion of the aneurysm and parent vessel with packing of the coils by an endovascular method. Some authors ${ }^{14}$ have reported that fusiform or dissecting aneurysms can be treated using endovascular methods.

\section{References:}

1. Al-Yamany M, Ross IB : Giant fusiform aneurysm of the middle cerebral artery : successful Hunterian ligation without distal bypass. Br J Neurosurg 12 : 572-575, 1998

2. Ceylan S, Karakus S, Duru S, Baykal S, Ilbay K : Reconstruction of the middle cerebral artery after excision of a giant fusiform aneurysm. Neurosurg Rev 21 : 189-193, 1998

3. Findlay JM, Hao C, Emery D : Non-atherosclerotic fusiform cerebral aneurysms. Can J Neurol Sci 29 : 41-48, 2002

4. Day AL, Gaposchkin CG, Yu CJ, Rivet DJ, Dacey RG Jr : Spontaneous fusiform middle cerebral artery aneurysms : characteristics and a proposed mechanism of formation. $\mathrm{J}$ Neurosurg 99 : 228-240, 2003

5. Park SH, MD; Yim MB, MD, PhD; Lee CY, MD; Kim E, MD; and Son EI,MD Intracranial Fusiform Aneurysms : $\mathrm{It}^{-}{ }^{-} \mathrm{s}$ Pathogenesis, Clinical Characteristics and Managements. JKNS 44(3):116-123,2008.

6. Echiverri HC, Rubino FA, Gupta SR, Gujrati M : Fusiform aneurysm of the vertebrobasilar arterial system. Stroke 20 : 1741-1747, 1989

7. Amagasaki K, Yagishita T, Yagi S, Kuroda K, Nishigaya K, Nukui H : Serial angiography and endovascular treatment of dissecting aneurysms of the anterior cerebral and vertebral arteries. J Neurosurg 91 : 682-686, 1999

8. Hashimoto H, Iida J, Shin Y, Hironaka Y, Sakaki T : Subarachnoid hemorrhage from intracranial dissecting aneurysms of the anterior circulation. Two case reports. Neurol Med Chir (Tokyo) 39 : 442-446, 1999

9. Lanzino G, Kaptain G, Kallmes DF, Dix JE, Kassell NF : Intracranial dissecting aneurysm causing subarachnoid hemorrhage : The role of computerized tomographic angiography and magnetic resonance angiography. Surg Neurol 48 : 477-481, 1997 
10. Nikawa S, Yamada J, Sumi Y, Yamakawa H : Dissecting aneurysm of the middle cerebral artery manifesting as subarachnoid hemorrhage and hemorrhagic infarctions. Case report. Neurol Med Chir (Tokyo) 42 : 62-66. 2002

11. Wakabayashi Y, Nakano T, Isono M, Shimomura T, Hori S : Dissecting aneurysm of the anterior cerebral artery requiring surgical treatment. Case report. Neurol Med Chir (Tokyo) 40 : 624-627, 2000

12. Chuang MJ, Lu CH, Cheng MH.Management of middle cerebral artery aneurysm. Asian J Surg 35(1):42-48,2012
13. Kurino M, Yoshioka S, Ushio Y : Spontaneous dissecting aneurysms of anterior and middle cerebral artery associated with brain infarction. A case report and review of the literature. Surg Neurol 57 : 428-437, 2002

14. Hoh BL, Putman CM, Budzik RF, Carter BS, Ogilvy CS : Combined surgical and endovascular techniques of flow alteration to treat fusiform and complex wide-necked intracranial aneurysms that are unsuitable for clipping or coil embolization. J Neurosurg 95 : 24-35, 2001 